\title{
Perforated Descending Aorta Treated by Graft Replacement in a Patient with Heart Failure
}

\author{
Mohammad Alsalaldeh*, Safak Simsek, Serkan Akcan, Mehmet Bozkurt and Halil Ibrahim Kutan \\ Department of Cardiovascular surgical, Faculty of Medicine Hospital, Pamukkale University, Turkey
}

*Corresponding author: Mohammad Alsalaldeh, Department of Cardiovascular surgical, Faculty of Medicine Hospital, Pamukkale University, Turkey

\begin{abstract}
Descending aortic pathologies carries a high mortality risk due to the location of the aorta and the difficulties of repair. An 83 years old male patient had been admitted via an emergency room with a chief complaint of upper abdominal pain and difficulty of breathing. Investigations showed a huge right hemi-thoracic hematoma associated with extravasation suggesting perforation of the distal descending aorta. TEVAR was planned but he was not suitable for TEVAR. Replacement of the descending aorta was performed successfully under general anesthesia and the use of a shunt from the proximal descending aorta to the abdominal aorta.
\end{abstract}

Keywords: Perforated Descending Aorta; Extrapleural Hematoma; Graft Bypass and Synthetic Shunt

\section{Introduction}

Descending aortic aneurysms, dissection or perforation are life-threatening pathologies. Elective open surgery for descending aortic aneurysm carries a high mortality risk up to $\% 10$, while emergent operations for acute dissection or rupture were found up to $41 \%$ [1]. In the last few decades, thoracic aortic endovascular repair (TEVAR) has been become the first treatment method in such cases [2]. Generally, aortic perforation occurs as a result of dissection or due to trauma or iatrogenic. In our case, the patient probably had perforation due to ulcerative plaque. He had a selflimited perforation in the distal part of the descending aorta. Firstly, we planned to manage this case with TEVAR but unfortunately, the descending aorta length, diameter, location of the perforation were not suitable for TEVAR. Also, the landing zone of the distal graft may occlude the celiac trunk by itself or by causing the mural thromboses to migrate into the celiac artery, so the decision was taken to operate the patient with an open surgical method.

\section{Case Report}

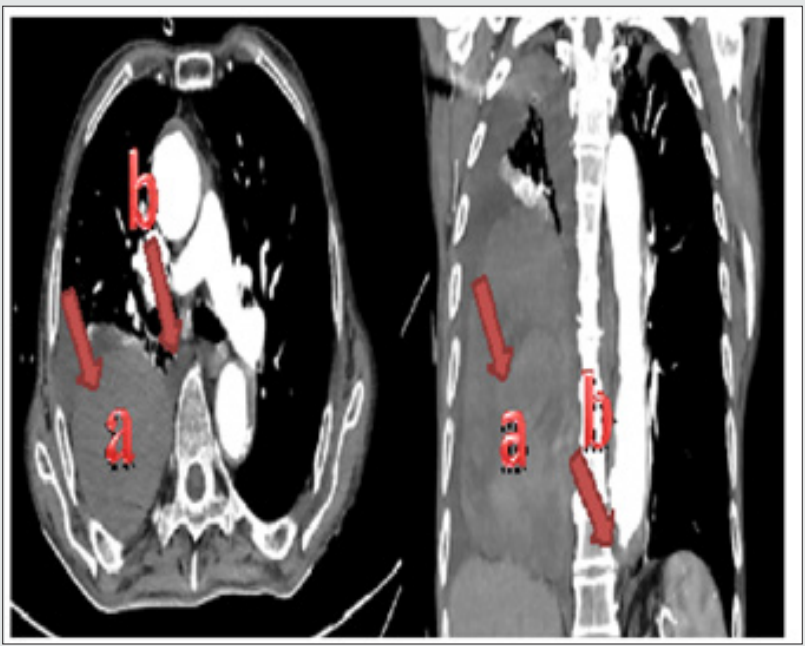

Figure 1: (a)CT-Scan with contrast showing the left-sided extrapleural hematoma, (b) the connection between the distal descending aorta and right hemithorax. 
An 83 years old male patient, known to have severe heart failure and renal impairment presented to our emergency room with chief complaints of upper abdominal pain, fatigue, the difficulty of breathing, and drowsiness. The patient's hemodynamics was unstable with low blood pressure and tachycardia. there was no abdominal rigidity or defense. Right lung sounds were diminished in the mid and lower zones. Blood investigation showed low hemoglobin and hematocrit. Thoracic and abdominal CT-Scan with contrast showed right extra pleural huge hematoma associated with dilated descending aortic mural thrombosis $(48.50 \mathrm{~mm})$ and extravasation to the right hemithorax (Figure 1). He was admitted to our intensive care unit (ICU) and a blood transfusion was done. TEVAR was planned, but unfortunately, it was not suitable for his case, that the perforation site is at the level of the diaphragm and the landing zone of the distal graft may occlude the celiac trunk by itself or by causing the mural thromboses to migrate into the celiac artery. We kept observing the patient for two days in the ICU, the average of blood transfusion was 2 units of erythrocytes suspensions and 2 units of frozen fresh plasma. When the patient's general condition became stable, a spinal drainage catheter was inserted, and he was taken to the operation room.

\section{Surgical Technique}

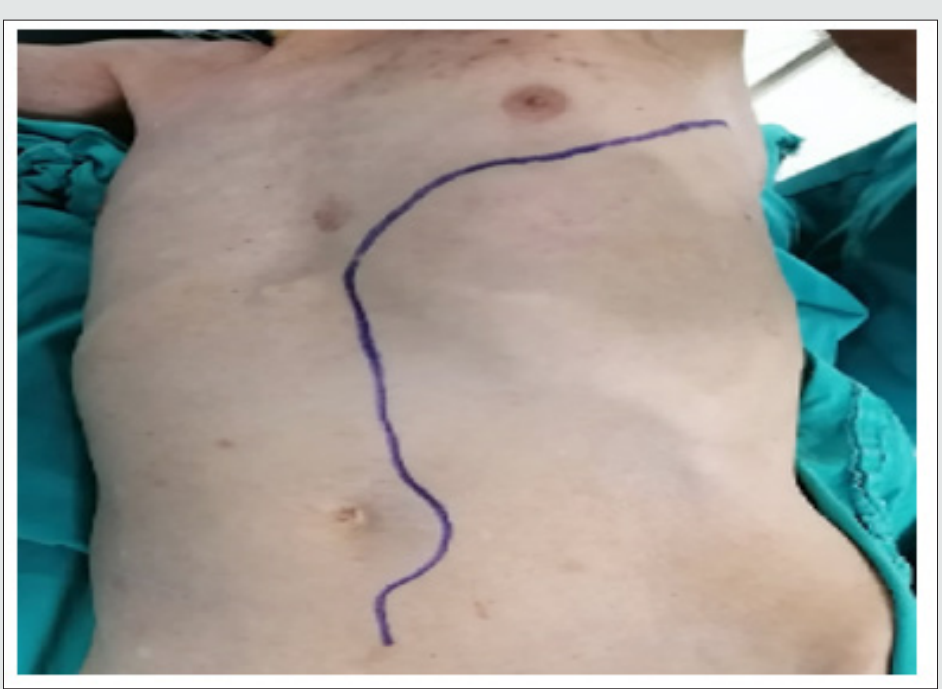

Figure 2: Incision site of the operation.

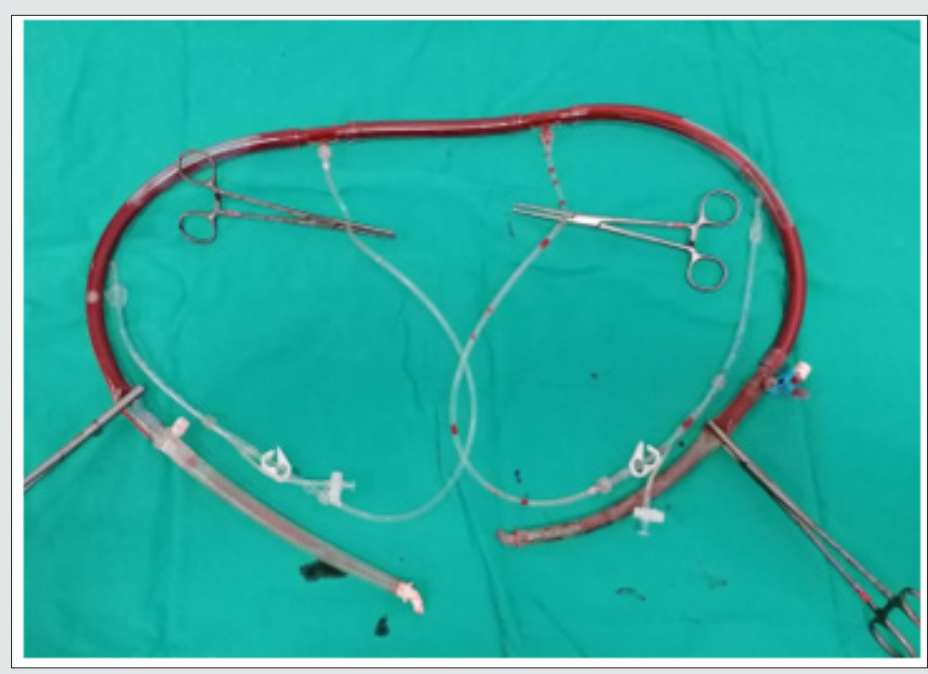

Figure 3: The shunt we used (with an arterial cannula at each side and two in the middle).

under general anesthesia and double-lumen tracheal intubation İnternal jugular vein was catheterized and a radial arterial line for blood pressure monitorization was inserted. The patient was put in the right lateral decubitus position with $60^{\circ}$ shoulders and $30^{\circ}$ hip flexion. The left arm was left up (Figure 2). After the skin preparation, a long incision was done starting from the lateral border of the left scapula through the 7.th intercostal space to the mid abdominal downward a few centimeters below the umbilicus. Thorax cavity was opened gently and attention was taken not to injure the left lung. The diaphragm was opened anterolaterally 
with leaving about 2 centimeters as a rim. The abdominal cavity was opened retroperitoneally. The thoracic aorta was reached. It was found dilated and calcific. The proximal and distal parts of the descending aorta at the level of the diaphragm were librated from the surrounding tissues and lift by vessel-loops after gentle dissection. Previously, we had prepared a shunt by using a piece from the tube that is used in a cardiopulmonary bypass kit of about $40 \mathrm{~cm}$ long. At each end of the tube, we connected an aortic cannula and at the middle of the tube, we connected 2 cardiac retrograde canulas (Figure 3). One side of the shunt was put at the proximal descending aorta while the other at the distal abdominal aorta and secured by double purse sutures.

The shunt was de-aired then its clamps were removed and the blood flow via the shunt started. A long vascular clamp was applied to the proximal of the descending aorta distally to the proximal shunt cannula and another clamp has applied to the end of the descending aorta at the level of the diaphragm. Aorta was opened longitudinally between the two clamps. The perforation site was found at the distal and medial sites of the thoracic aorta, a few centimeters above the diaphragm level of about 2-2.5 cm in diameter (Figure 4). It was occluded by a thrombus. Spinal arteries orifices were all thrombosed, so there was no need to be canulated to perform anastomosis to the graft laterally. When the thrombus was removed blood flow started coming from the right hemithorax. The perforation site was enlarged by the surgeon's fingers and the hematoma and thrombosis were evacuated by the hand of the surgeon and by using an aspirator. $30 \mathrm{~mm}$ Dacron graft was anastomosed to the proximal side of the descending aorta distally to the clamp then proximal clamp removed from the proximal descending aorta and applied to the graft distally to the anastomosis site. The distal part of the graft was anastomosed to the distal part of the descending aorta and proximally to the vascular clamp. The distal clamp was removed after de-airing and the hemostasis was secured (Figure 5).

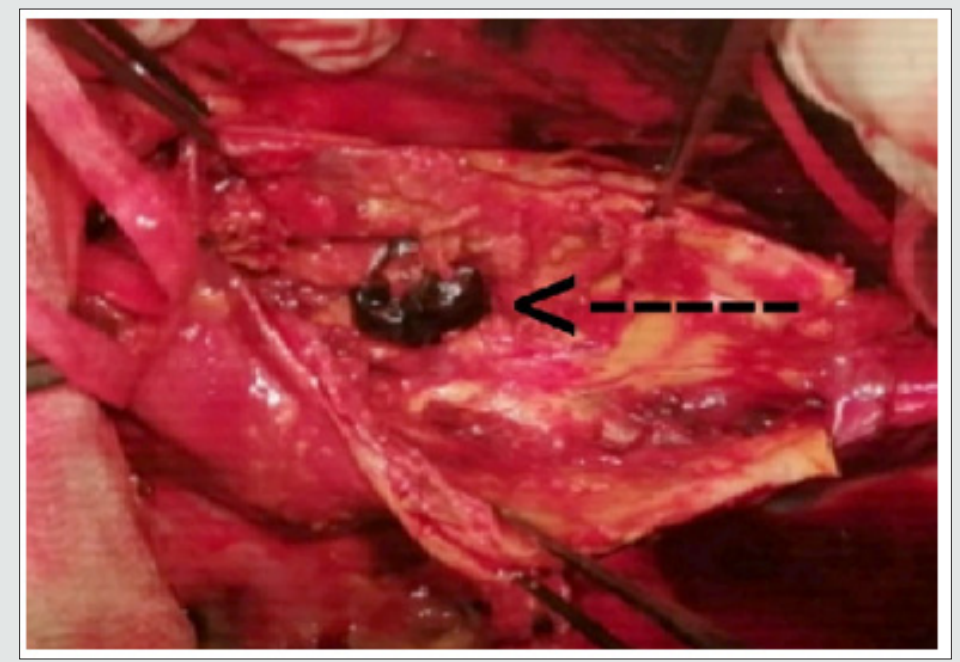

Figure 4: The perforation site of the distal ascending aorta.

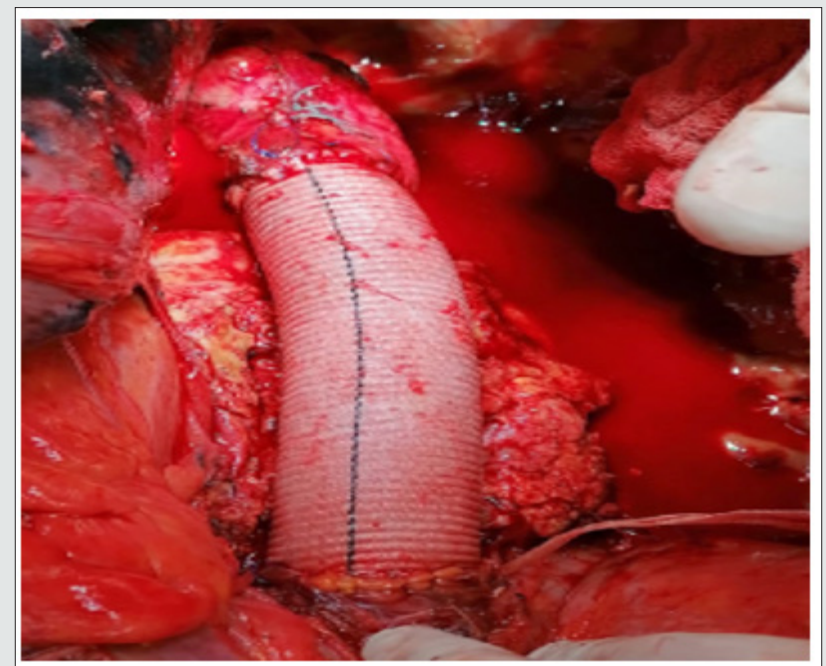

Figure 5: $30 \mathrm{~mm}$ Dacron graft after had been anastomosed to the proximal and distal descending aorta site. 
The shunt was clamped on both sides and removed. Purse sutures were tied. The opened sac of the aorta was closed over the graft. Two chest tubes were inserted into the left hemithorax where one was inserted into the right. Two soft drains were inserted into the abdomen. Chest and abdominal walls were closed in the fashion way after suturing the diaphragm from where it was cut. The patient was taken to the ICU for closed observation and medical treatment. On the 2.nd operative day, he was extubated. There was not any neurological deficit and the blood tests were at acceptable levels. On the first operative day, the total drainage was about 450 and decreased over time. Two units of erythrocytes suspension and two fresh frozen plasma transfusion was done. Drains were removed on the fourth day postoperatively and the patient was transferred to the floor on the 5.th day. Daily dressing and investigation were done on the floor. On the 10.th day, he was discharged to be seen after one week as an outpatient. The patient has been followed up for three months. He is free from any complaints and controls Ct-scan and blood investigations are in acceptable ranges.

\section{Discussion}

Descending aortic perforation is one of the most critical pathologies of the aorta usually treated by TEVAR when it's possible. Open surgical repair carries high morbidity and mortality risk and should be kept as the last treatment choice. In our case, we tried to do TEVAR because it has less morbidity and mortality than open surgery but unfortunately, our patient was not fit for TEVAR, so the decision was taken to do open surgery after discussing all the possible complications with the patient's relatives. In thoracoabdominal aortic surgery usually left heart bypass is used to maintain the blood flow to the visceral organs [3]. In this case and because the patient had severe heart failure we preferred to do a synthetic vascular shunt and not to touch the heart and it was successful. We designed a shunt ourselves by using a tube from the cardiopulmonary kit and connected an aortic cannula to each end of the tube. In the middle, we put a connector with two 3-way stop taps that connected to 4 small cannulas (we used the retrograde cardioplegic cannulas) to secure the blood flow to the spinal artery if needed. When the descending aorta was opened all spinal arteries orifices were founded occluded and thrombosed so those four cannulas were not used.

On the other hand, we faced a problem with the ventilation of the lungs, the right lung could not be ventilated enough due to the extrapleural hematoma while the left lung was inflated and deflated intermittently to give us chance to work which increased the time of operation that we couldn't work while the left lung is inflated. The total time of the operation was 5 hours. The clamping time was about 45 minutes. Spinal drainage perioperatively played a role in decreasing the possibility of having neurological deficit postoperatively [4]. A spinal cerebrospinal fluid drainage catheter was inserted at the night before the operation and removed on postoperative 1.st day. There was no neurological deficit and the patient could remove all his extremities. If the spinal arteries were not occluded we would maintain their blood flow by using our prepared shunt canulas, then anastomosed them to the graft, but when they were found occluded we did not need to use those cannulas or to do anastomosis.

\section{Conclusion}

When a decision is taken to do open surgery for descending aortic repair, good planning should be considered as the cornerstone. The patient's general conditions and his co-exist morbidity should be kept in mind too. Even in the perforated descending aorta and multimorbidity cases the operation can be carried out well and done successfully with minimal complications if the spinal cord drainage and visceral organs perfusion can be maintained during the operation [5]. As well as the left heart bypass can be used for visceral organ perfusion a well-branched synthetic shunt from the proximal descending aorta to the abdominal aorta or iliac artery can maintain blood flow to the visceral organs too. Informed consent has been obtained from the patient and his family for publication of the case report and accompanying images.

\section{Declaration of Conflicting Interests}

All the authors declared no conflicts of interest concerning the authorship and/or publication of this article.

\section{Funding}

The authors received no financial support for the research and/ or authorship of this article.

\section{References}

1. Fischbein MP, Miller DC (2009) Long-term durability of open thoracic and thoracoabdominal aneurysm repair. Semin Vasc Surg 22(2): 74-80.

2. Grabenwöger M, Alfonso F, Bachet J, Bonser R, Czerny M, et al (2012) Thoracic Endovascular Aortic Repair (TEVAR) for the treatment of aortic diseases: a position statement from the European Association for CardioThoracic Surgery (EACTS) and the European Society of Cardiology (ESC), in collaboration with the European Association of Percutaneous Cardiovascular Interventions (EAPCI). European heart journal 33(13): 1558-1563.

3. Schepens MA (2016) Left heart bypass for thoracoabdominal aortic aneurysm repair: technical aspects. Multimedia manual of cardiothoracic surgery: MMCTS.

4. Khan NR, Smalley Z, Nesvick CL, Lee SL, Michael LM(2016) The use of lumbar drains in preventing spinal cord injury following thoracoabdominal aortic aneurysm repair: An updated systematic review and meta-analysis. Journal of Neurosurgery: Spine 25(3): 383393.

5. Wong DR, Lemaire SA, Coselli JS(2008) Managing dissections of the thoracic aorta. The American surgeon 74(5): 364-380. 
CC (P) This work is licensed under Creative

To Submit Your Article Click Here: Submit Article

DOI: $10.32474 /$ SCSOAJ.2021.06.000238

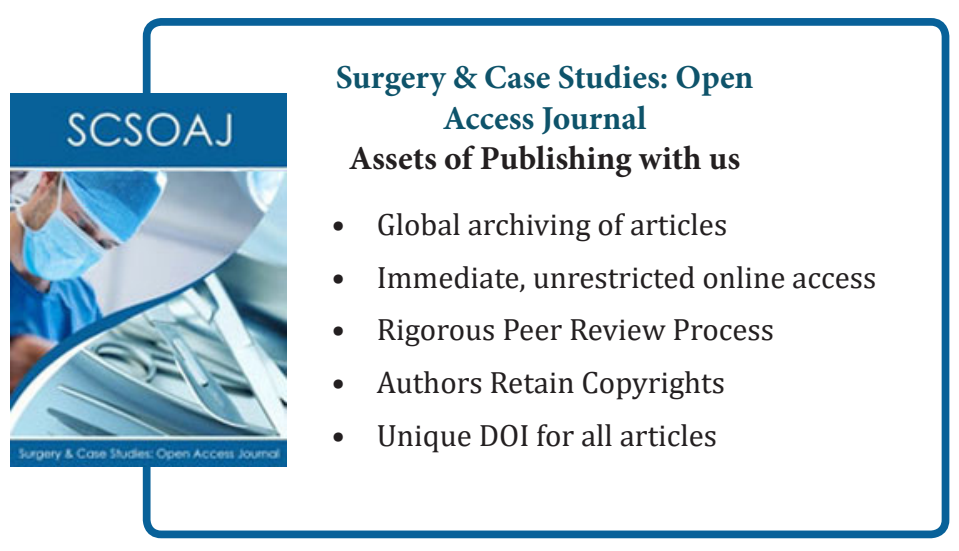

